\title{
RELATOS DE EXPERIÊNCIA \\ PERSPECTIVAS DE PESQUISA SOBRE INTELIGÊNCIA ESTRATÉGICA ANTECIPATIVA E COLETIVA (IEAC) POR MEIO DA ANÁLISE DE SENTIMENTO: UM CENÁRIO DIDÁTICO DE USO
}

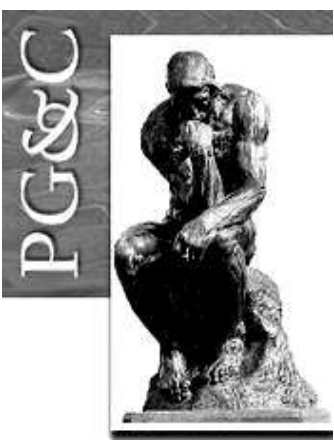

\author{
Guilherme Bertoni Machado \\ Doutorando em Engenharia e Gestão do Conhecimento pela Universidade \\ Federal de Santa Catarina, Brasil. Professor da Faculdade Senac Porto \\ Alegre e da Escola Superior de Propaganda e Marketing, Brasil. \\ E-mail: gbertonimachado@gmail.com \\ Rodrigo Kraemer \\ Doutorando em Engenharia e Gestão do Conhecimento pela Universidade \\ Federal de Santa Catarina, Brasil. Policial Rodoviário Federal, Brasil. \\ E-mail: rodrigokop@gmail.com

\section{Gertrudes Aparecida Dandolini} \\ Doutora em Engenharia de Produção pela Universidade Federal de Santa \\ Catarina, Brasil. Professora da Universidade Federal de Santa Catarina, \\ Brasil. \\ E-mail: gtude@egc.ufsc.br

\section{João Artur de Souza} \\ Doutor em Engenharia de Produção pela Universidade Federal de Santa \\ Catarina, Brasil. Professor da Universidade Federal de Santa Catarina, \\ Brasil. \\ E-mail: jartur@egc.ufsc.br

\section{José Leomar Todesco} \\ Doutor em Engenharia de Produção pela Universidade Federal de Santa \\ Catarina, Brasil. Professor da Universidade Federal de Santa Catarina, \\ Brasil. \\ E-mail: jose.todesco@ufsc.br
}

\begin{abstract}
Resumo
Estrategicamente as empresas devem estar em constante capacidade de adaptação, ou seja, atentas aos movimentos externos em relação a eventos futuros. Portanto, há a necessidade da exploração de sinais fracos, através da detecção, interpretação e uso desses sinais para antecipação. O objetivo deste relato de experiência é apresentar perspectivas de pesquisa sobre Inteligência Estratégica Antecipativa e Coletiva (IEAc) por meio da Análise de Sentimento. Dentro deste contexto, e seguindo o método pesquisa-ação, foi proposto e implementado através da ferramenta Node-RED da IBM, um cenário didático de uso da análise de sentimento. O experimento consistiu no monitoramento de posts no Twitter sobre um determinado assunto indexado através de uma hashtag. Caso o post fosse analisado pelo algoritmo de análise de sentimento como negativo, um e-mail é enviado para um determinado usuário para que este faça a gestão/tomada de decisão sobre o assunto. Este cenário de uso foi aplicado para a demonstração desta técnica de Advanced Analytics com o intuito de trazer uma forma inovadora para a IEAc.
\end{abstract}

Palavras-chave: Inteligência Estratégica Antecipativa e Coletiva. Advanced Analytics. Análise de Sentimento. Monitoramento do Ambiente.

Perspectivas em Gestão \& Conhecimento, João Pessoa, v. 10, n. 1, p. 152-164, jan./abr. 2020. DOI: http://dx.doi.org/10.21714/2236-417X2020v10n1p152

http://periodicos.ufpb.br/ojs2/index.php/pgc. ISSN: 2236-417X. Publicação sob Licença (cc) EY-NC-ND 


\title{
RESEARCH PERSPECTIVES ON ANTECIPATIVE AND COLECTIVE STRATEGIC INTELLIGENCE (IEAC) THROUGH SENTIMENT ANALYSIS: A DIDACTIC USE SCENARIO
}

\begin{abstract}
Strategically companies must be in constant adaptability, in other words, watchful to external movements regarding future events. So, there is the need of weak signals exploration through the detection, interpretation and use of these signals to anticipation. This experience report aims to present research perspectives on Anticipative and Collective Strategic Intelligence (IEAc) through Sentiment Analysis. Within this context, and following the action research method, it was proposed an implemented through IBM's Node-RED tool, an educational scenario of sentiment analysis use. The experiment consisted in monitoring Twitter posts on a determinate subject indexed through a hashtag. If the analyzed post was considered negative by sentiment analysis algorithm, an e-mail is sent to a determinate user so this user can do the management/decision making on the subject. This scenario is applied for demonstration of this Advanced Analytics technique in order to bring an innovative way to IEAc.
\end{abstract}

Keywords: Anticipative and Collective Strategic Intelligence. Advanced Analytics. Sentiment Analysis. Environmental Scanning.

\section{INTRODUÇÃO}

Estrategicamente as empresas devem estar em constante capacidade de adaptação, ou seja, atentas aos movimentos externos em relação a eventos futuros. Portanto, há a necessidade da exploração de sinais fracos, através da detecção, interpretação e uso desses sinais para antecipação.

O que diferencia as empresas em mercados altamente competitivos é a capacidade de tomar decisões precisas, oportunas e eficazes, em todos os níveis - operacional, tático e estratégico, para atender as preferências de seus clientes (BOSE, 2009).

Para conseguir tomar decisões nesse nível as empresas estão utilizando advanced analytics para analisar seus dados, combinando informações sobre circunstâncias passadas, eventos atuais ou projeções futuras (BOSE, 2009). Embora muitas empresas estejam adotando a análise (analytics), poucas tem alcançado um bom nível de proficiência estando prontas para competir nessa área (DAVENPORT, 2006).

Advanced analytics é considerado um termo geral que significa simplesmente aplicar várias técnicas analíticas avançadas nos dados para responder a perguntas ou resolver problemas, não sendo uma tecnologia em si, mas um grupo de ferramentas que combinadas possibilitam obter informação, analisar e prever resultados (BOSE, 2009).

A base da Advanced analytics é a mineração de dados, a mineração de texto e a mineração web. Mineração de dados é utilizada para extração automática de padrões, associações, alterações, anomalias e estruturas significantes de dados; a mineração de texto aplica as mesmas funções analíticas de mineração de dados para as informações textuais; e a mineração web que é uma forma de mineração para descobrir padrões web (BOSE, 2009).

Conforme Pang e Lee (2008 apud Chen, Chiang e Storey, 2012, p. 1176), "análise de sentimento é geralmente utilizada na mineração de opinião para identificação de sentimento, afeição, subjetividade e outros estados emocionais em textos online. A Web 2.0 e o conteúdo das mídias sociais têm criado oportunidades abundantes e excitantes para o entendimento das opiniões de consumidores e público em geral em relação a eventos sociais, movimentos políticos, estratégias de empresas, campanhas de marketing e preferências de produtos.".

Perspectivas em Gestão \& Conhecimento, João Pessoa, v. 10, n. 1, p. 152-164, jan./abr. 2020. 
Segundo Janissek-Muniz, Lesca e Freitas (2007) "o que é uma informação tipo sinal fraco e como saber se a informação é antecipativa e portadora de sentido útil para os tomadores de decisão?". Em cima desta pergunta é possível buscar estudos de como os trabalhadores do conhecimento usam a Web para pesquisar informações externas que fazem parte do seu trabalho diário. Análise de sentimento foi identificado por Lim, Chen e Chen (2013) como uma direção de pesquisa.

Um ambiente de Inteligência Estratégica Antecipativa e Coletiva (IEAc) estimula as organizações a realizarem tarefas de análise, síntese e avaliação. Nesse sentido, as estratégias que promovem IEAc podem ser definidas como sendo atividades que ocupam as organizações em entender melhor o seu negócio e o mercado.

O objetivo deste relato de experiência é apresentar perspectivas de pesquisa sobre IEAc por meio da Análise de Sentimento. Um cenário de uso foi aplicado para a demonstração desta técnica de Advanced Analytics com o intuito de trazer uma forma inovadora para a IEAc.

O experimento consistiu no monitoramento de posts no Twitter sobre um determinado assunto indexado através de uma hashtag. Caso o post fosse analisado pelo algoritmo de análise de sentimento como negativo, um e-mail é enviado para um determinado usuário para que este faça a gestão/tomada de decisão sobre o assunto.

Este relato contém 5 seções: a introdução apresenta a teoria de base e a pergunta de pesquisa utilizada no desenvolvimento deste relato. A seção 2 apresenta a fundamentação teórica conceituando o que autores falaram sobre os assuntos abordados, oferecendo assim a possibilidade, na seção 3 , da definição do método utilizado e da descrição do experimento realizado na seção 4 . Por fim, temos na seção 5 as considerações finais deste ensaio.

\section{FUNDAMENTAÇÃO TEÓRICA}

Foi Schumpeter (1961) quem primeiro diferenciou invenção de inovação, afirmando que esta é intimamente associada à ideia de aplicação. Assim, se a invenção é mais teórica ou abstrata, a inovação se utiliza da invenção e a coloca em prática. O Manual de Oslo apresenta a inovação como a implementação de algo novo ou melhorado, sendo de quatro tipos: inovação de produto, inovação de processo, inovação de marketing e inovação organizacional (MANUAL DE OSLO, 2004).

A inovação pode ser definida como todo novo conhecimento incorporado em produtos, processo ou serviços (POPADIUK; CHOO, 2006). Mas além de novidade, o importante na inovação está na capacidade de agregar valor aos produtos ou serviço de uma organização diferenciando-a no ambiente competitivo (BAUTZER, 2000).

Para Morris (2009), a inovação é um processo e também o resultado do processo. Enquanto processo deve ser gerido, ou seja, ter ações organizacionais para que alcance resultados inovadores. Tidd, Bessant e Pavitt $(2008)$ reforçam a importância do gerenciamento da inovação pois ela só existe depois do sucesso, o que existe antes é o processo e é isto que deve ser gerenciado.

Inovação não é uma ação única, mas um processo total de subprocessos interrelacionados. Não é apenas a concepção de uma ideia nova, nem a invenção de um novo dispositivo, nem o desenvolvimento de um novo mercado. O processo consiste de todas essas coisas agindo de forma integrada (TROTT, 2012).

A capacidade de inovação continua está associada ao Processo de Gestão do Conhecimento criado durante o processo de inovação, o conhecimento gerado é a essência para um novo processo de inovação (QUINTANE et al., 2011; XU et al., 2010).

$\mathrm{O}$ embasamento teórico deste relato de experiência foi concebido para auxiliar a um melhor entendimento da pergunta de pesquisa deste trabalho: como prover a Inteligência Estratégica Antecipativa e Coletiva por meio da Análise de Sentimento?

Perspectivas em Gestão \& Conhecimento, João Pessoa, v. 10, n. 1, p. 152-164, jan./abr. 2020. 
Através desta pergunta foi possível ter um 'produto' de pesquisa, que é o objetivo do relato, ou seja, apresentar um cenário didático que mostra a aplicação da análise de sentimento com o intuito de trazer uma forma inovadora para a Inteligência Estratégica Antecipativa e Coletiva.

Portanto, se faz necessário aprofundar sobre Inteligência Estratégica Antecipativa e Coletiva e Análise de Sentimento, que serviram como arcabouço teórico, dando suporte à ideia do experimental realizado.

O aprofundamento sobre os temas deste relato de experiência está descrito nas subseções 2.1 e 2.2 e foram realizados através de uma revisão narrativa.

\subsection{Inteligência Estratégica Antecipativa e Coletiva (IEAc)}

A Inteligência Competitiva (IC) é um processo sistemático que transforma dados e partes de informações em conhecimento estratégico para tomada de decisão (TYSON, 1998). O benefício mais comum da inteligência competitiva é a capacidade para construir perfis de informação que ajuda a organização a identificar os seus concorrentes, os pontos fracos e fortes, estratégias, objetivos, posicionamento de mercado e padrões de reação prováveis (BOSE, 2008).

O processo de IC é a ação de coleta, análise e utilização de informações sobre os produtos, concorrentes, fornecedores, reguladores, parceiros e clientes para as necessidades de planejamento de uma organização (KAHANER, 1997). O processo pode ser descrito em fases cíclicas como planejamento, pesquisa, análise, disseminação e feedback (BOSE, 2008, p. 513).

Conforme Lesca e Janissek-Muniz (2015), "Os sinais são identificados através do monitoramento do ambiente pertinente à empresa, o qual visa fornecer informações voltadas ao futuro para que a organização possa estruturar-se de forma precoce contra as ameaças e alavancar as novas oportunidades, ambas ligadas aos atores do ambiente em que está inserida.".

Ainda conforme Janissek-Muniz, Lesca e Freitas (2011, p. 103) a "IEAc é uma proposta que visa auxiliar as organizações na sua capacidade em antecipar alterações de seu ambiente sócio-econômico, e a considerá-las na definição dos eixos estratégicos que ela deseja implantar. Para tal, as organizações devem ser capazes (ou serem capacitadas a) de coletar, selecionar e analisar informações relativas ao estado e à evolução do ambiente onde elas se encontram. A implantação de um sistema de monitoramento do ambiente é o que chamamos de IEAc.".

\subsection{Análise de Sentimento}

Conforme Dos Santos (2018), "o campo de pesquisa da análise de sentimento (sentiment analysis), também conhecido como mineração de opinião (opinion mining), tem crescido, nos últimos anos, impulsionado, basicamente, pela difusão das plataformas de mídias sociais, pela importância das mesmas como ambientes de sociabilidade e comunicação, bem como as diversas aplicações possíveis desse tipo de estudo, incluindo o marketing (de marcas, lugares, político), eleições, análise de mercados e até índices de ações negociadas em bolsa, associados à percepção das pessoas sobre suas tendências de crescimento ou queda".

De acordo com Silva, Lima e Barros (2012), "na última década, pudemos testemunhar o rápido crescimento de Blogs, Fóruns e Redes sociais na Web. Milhões usuários tornam disponíveis suas opiniões a respeito dos mais diversos assuntos por meio desses sites e ferramentas. Esse tipo de informação é de grande importância como auxílio na tomada de decisão, tanto para empresas, como para usuários comuns. Contudo, essas opiniões estão

Perspectivas em Gestão \& Conhecimento, João Pessoa, v. 10, n. 1, p. 152-164, jan./abr. 2020. 
dispersas na Web, em formato livre, tornando impraticável a busca e análises manuais dessa informação.".

Ainda segundo Silva, Lima e Barros (2012), "nesse contexto, surgiram sistemas para tratar opiniões automaticamente, utilizando-se dos conceitos da área de Análise de Sentimentos (AS), também conhecida como Mineração de Opiniões. A AS, dentre outras tarefas, preocupa-se em classificar opiniões expressas em textos a respeito de um determinado objeto (produto, serviço, instituição ou pessoa) como positivas (e.g., a pizza estava ótima (+)), ou negativas (e.g., a cerveja estava quente (-)). Neste caso, a classificação é feita com base nos adjetivos encontrados no texto, também chamados de palavras opinativas.".

A Figura 1 ilustra a arquitetura geral para o processo de uma análise de sentimento genérica:

Figura 1 - Arquitetura geral para o processo de uma análise de sentimento genérica

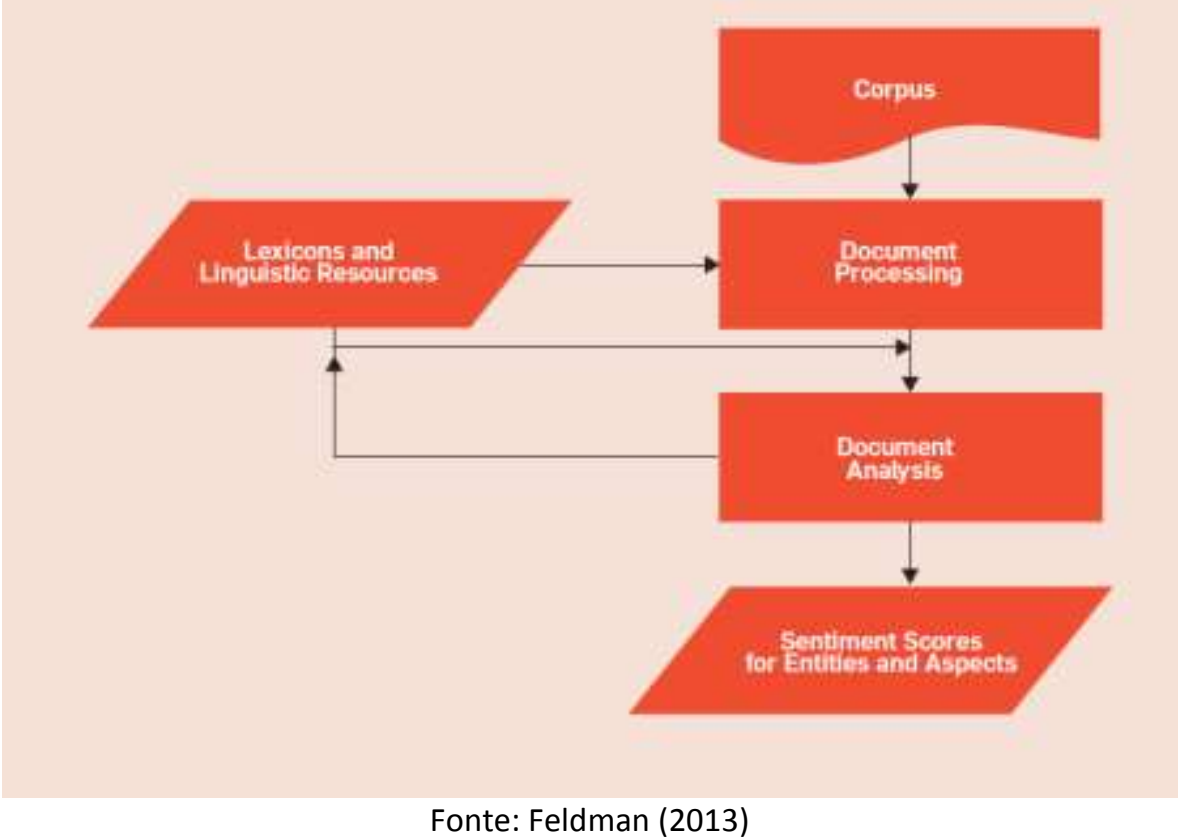

A entrada do sistema é composta de um corpus em algum formato (PDF, HTML, XML, etc) que é convertido em texto plano. Este texto (documento) é processado usando técnicas de mineração de texto baseadas em ferramentas linguísticas, como tokenização, stemming, etc. $O$ sistema também pode usar algum módulo que utilize léxicos e recursos linguísticos para melhorar o processamento e a análise do documento. O módulo de análise do documento irá realizar as anotações de sentimentos que podem ser vinculadas ao documento como um todo, ou as sentenças individuais ou para aspectos específicos de determinada entidade. A saída do sistema pode ser apresentada em uma ferramenta de visualização (FELDMAN, 2013).

\section{PERSPECTIVAS DE PESQUISA SOBRE INTELIGÊNCIA ESTRATÉGICA ANTECIPATIVA E COLETIVA POR MEIO DA ANÁLISE DE SENTIMENTO}

Segundo Janissek-Muniz, Lesca e Freitas (2011, p. 104), a "IEAc busca, através de métodos próprios, fornecer uma representação do ambiente pertinente à empresa, e elementos que permitam inferir mudanças desse ambiente, visando apoiar o processo decisório organizacional e agir de forma rápida, no momento certo.". 
De acordo com Chen, Chiang e Storey (2012, p. 1167), “Além de capturar a popularidade de uma celebridade, as referências de eventos cotidianos e sentimentos sociopolíticos expressos nestes meios de comunicação, aplicações Web 2.0 pode eficientemente reunir um grande volume de feedback em tempo útil e opiniões de uma população diversificada dos clientes para diferentes tipos de empresas.".

Lim, Chen e Chen utilizam o termo Business Intelligence and Analytics - BIA e a define como tecnologias, sistemas, práticas e aplicações que analisam dados críticos de negócios para ajudar uma empresa a entender melhor o seu negócio e o mercado (LIM; CHEN; CHEN, 2013).

Portanto partindo das premissas citadas nos parágrafos anteriores, esta seção apresenta uma proposta didática para inteligência estratégica antecipativa e coletiva por meio da análise de sentimento.

\subsection{Metodologia proposta}

Para que se possa realizar uma pesquisa empírica, ou seja, que se obtenha o conhecimento através da observação do objeto de estudo é necessário que esta pesquisa tenha uma finalidade e um método científico. A finalidade está relacionada aos objetivos, isto é, ter claramente definido o que se está buscando obter com a pesquisa. Já o método científico diz respeito ao modo como os resultados são obtidos (TRIPODI et al. apud OLIVEIRA; MAÇADA; GOLDONI, 2006).

A estratégia de averiguação acerca da Inteligência Estratégica Antecipativa e Coletiva através da Análise de Sentimento foi realizada por meio de um experimento investigativo de caráter qualitativo e dedutivo baseado no método pesquisa-ação (THIOLLENT, 2011) sendo este experimento idealizado e realizado pelos autores deste relato de experiência, utilizando uma ferramenta open source disponibilizada pela IBM denominada Node-Red.

O interesse neste tipo de estratégia de pesquisa aumentou devido à insatisfação com as técnicas quantitativas uma vez que estas apresentam as seguintes desvantagens (BENBASAT; MEAD, GOLDSTEIN, 1987):

a) Complexidade de pesquisas multivariadas;

b) Restrições da distribuição inerentes ao uso destes métodos (normalidade multivariada);

c) Grandes amostras;

d) Dificuldade de entender e interpretar os resultados dos estudos em que métodos quantitativos complexos são utilizados.

\subsection{Apoio a Inteligência Estratégica Antecipativa e Coletiva}

Conforme Janissek-Muniz, Lesca e Freitas (2011, p. 106), o "caráter antecipativo de uma informação não significa antecipação no sentido de capacidade de previsão nem de tendência. Previsão e tendência, muitas vezes, suscitam a idéia de analisar o passado para prever o futuro, supondo que haverá uma continuidade de comportamentos. O caráter antecipativo aqui considerado diz respeito à inovação. Muitas vezes, pequenos fragmentos de informações podem conter sementes de inovação, que podem vir eventualmente a significar uma grande ruptura.".

Com o surgimento da web 2.0 uma quantidade imensa de informações pode ser conseguida da empresa e do consumidor, não apenas em banco de dados internos, mas também através da web.nos adjetivos encontrados no texto, também chamados de palavras opinativas.".

A mineração web pode se subdividir em (BOSE, 2009):

Perspectivas em Gestão \& Conhecimento, João Pessoa, v. 10, n. 1, p. 152-164, jan./abr. 2020. 
a) Mineração de conteúdo web: extrair conhecimento através do conteúdo web;

b) Mineração de estrutura web: ligações entre as referências da web;

c) Mineração de uso da web (ou mineração de web log): analisa o comportamento do usuário no site para extrair padrões interessantes, podendo ser usados para fornecer métricas sobre a eficácia do site web ou o sucesso de uma campanha em particular.

Logo, considerando os pontos citados nos parágrafos anteriores, foi criado um cenário de utilização em uma rede social que irá simular as opiniões de usuários a respeito de uma determinada instituição (que é indexada através de uma hashtag).

Assim os gestores desta instituição podem receber feedback (através da análise de sentimento dos posts feitos por estes usuários) sobre os seus produtos e tomar decisões em tempo real se determinado usuário não está contente.

\section{APLICAÇÃO}

Dentro do contexto apresentado ao longo do relato de experiência, e seguindo o método pesquisa-ação, foi proposto e implementado através da ferramenta Node-RED da IBM, um cenário didático de uso da análise de sentimento. O experimento consistiu no monitoramento de posts no Twitter sobre um determinado assunto (no caso qualquer menção ao tópico \#egc) indexado através de uma hashtag.

Caso o post fosse analisado pelo algoritmo de análise de sentimento como negativo (ou seja, algum usuário/cliente está insatisfeito com um produto e/ou serviço prestado pela instituição ou pessoa referenciada pela hashtag), um e-mail é enviado para um determinado usuário para que este faça a gestão/tomada de decisão sobre o assunto.

O Node-RED ${ }^{1}$ é uma ferramenta visual que é executada em qualquer navegador internet. Utiliza o Node.js ${ }^{2}$ como servidor de aplicação e tem uma série de recursos para conexão de dispositivos de hardware, APIs $^{3}$ e serviços online. Já possui nativamente a implementação do algoritmo de análise de sentimento, o que facilita o uso.

Este algoritmo de análise de sentimento é denominado "AFINN-based sentiment analysis for Node.js" proposto por Hansen et al. (2011), sendo que a última versão é a AFINN111 que tem 2477 palavras e frases em sua base de conhecimento, sendo os valores típicos de análise vão de $-5 a+5$ (maiores que zero são positivos e menores que zero são negativos), mas podem ser mais baixos ou mais altos. Um detalhe importante é que o algoritmo só trabalha com palavras na língua inglesa.

\subsection{Cenário de Uso}

O cenário de uso segue a estrutura ilustrada pela Figura 2. Uma vez implantado este cenário, a ferramenta irá procurar os tweets referenciados através de uma hashtag determinada, sendo processados para a geração do corpus que será analisado pelo algoritmo de análise de sentimento.

Se o valor for fortemente negativo (menor ou igual a -5) este tweet será enviado ao administrador da instituição/pessoa referenciada negativamente para que a mesma possa tomar uma decisão e fazer a gestão da marca/serviço e do produto. No mesmo ambiente uma

\footnotetext{
${ }^{1}$ Disponível em: http://nodered.org/.

${ }^{2}$ Node.js é um interpretador de código JavaScript que funciona do lado do servidor.

3 API provém do Inglês Application Programming Interface, é um conjunto de rotinas e padrões estabelecidos por um software para a utilização das suas funcionalidades por aplicativos que não pretendem envolver-se em detalhes da implementação do software, mas apenas usar seus serviços.
}

Perspectivas em Gestão \& Conhecimento, João Pessoa, v. 10, n. 1, p. 152-164, jan./abr. 2020. 
saída de teste foi criada para que se pudessem ver todos os valores de sentimento atribuídos aos tweets, tanto os positivos quanto os negativos.

Figura 2 - Cenário de Uso

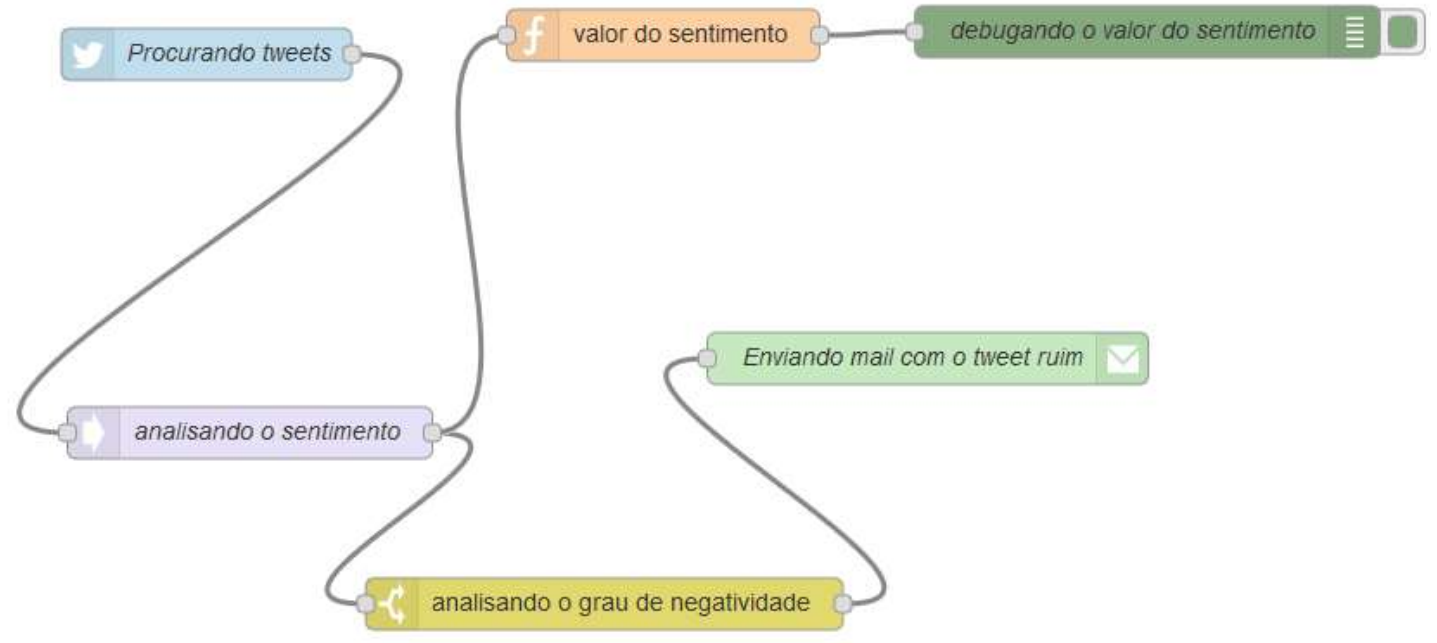

Fonte: Autores do relato

A Figura 3 apresenta a configuração para que se possa realizar a conexão com a API do Twitter para que a ferramenta possa a receber os tweets que correspondem ao critério de busca (que no caso serão todos os tweets que tiverem a hashtag EGC, ou seja, tenham no seu conjunto de caracteres \#egc).

Figura 3 - Configuração com a API do Twitter

\section{Edit twitter in node}

- Twitter ID

@bertoniMachado

Q Search

for

Name all public tweets

\#egc

Procurando tweets

Fonte: Autores do relato

A Figura 4 apresenta a configuração para que se possa definir qual o parâmetro deve desencadear alguma ação após a análise de sentimento. Neste caso, se o valor da análise de sentimento for menor ou igual a -5 do post processado com a hashtag definida como critério de busca (lembrando que o post deve ser em inglês por uma limitação do algoritmo) a ferramenta irá executar o módulo de envio de e-mail, ilustrado pela Figura 5: 
Figura 4 - Configuração de parâmetro para análise do grau de negatividade Edit switch node

Name

analisando o grau de negatividade

Property msg. sentiment.score

$<=$

$-5$

$\rightarrow 1$

Fonte: Autores do relato

O post completo será enviado para a caixa de mail definida na configuração, assim será possível entrar em contato com o usuário insatisfeito, bem como apenas monitorar o ambiente.

Figura 5 - Configuração de envio de mail

\section{Edit e-mail node}

To

$$
\text { gbertonimachado@gmail.com }
$$

Server

smtp.gmail.com

$x$ Port

$$
465
$$

Userid

gbertonimachado@gmail.com

Password

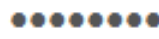

Enviando mail com o tweet ruim

Fonte: Autores do relato

A Figura 6 apresenta a configuração para a saída de teste do ambiente, ou seja, todos os posts são analisados e apresentados na ferramenta com o seu respectivo valor de sentimento atribuído, conforme Figura 7:

Perspectivas em Gestão \& Conhecimento, João Pessoa, v. 10, n. 1, p. 152-164, jan./abr. 2020. 
Figura 6 - Saída de teste

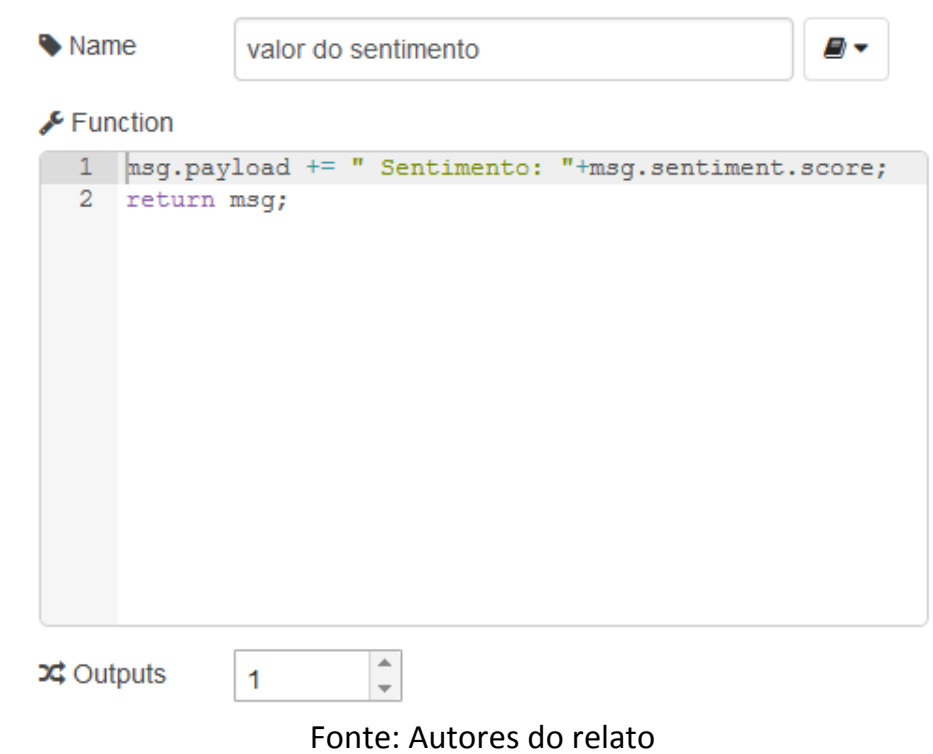

O post completo apresentado na área de debug concatenado com o termo Sentimento: valor.

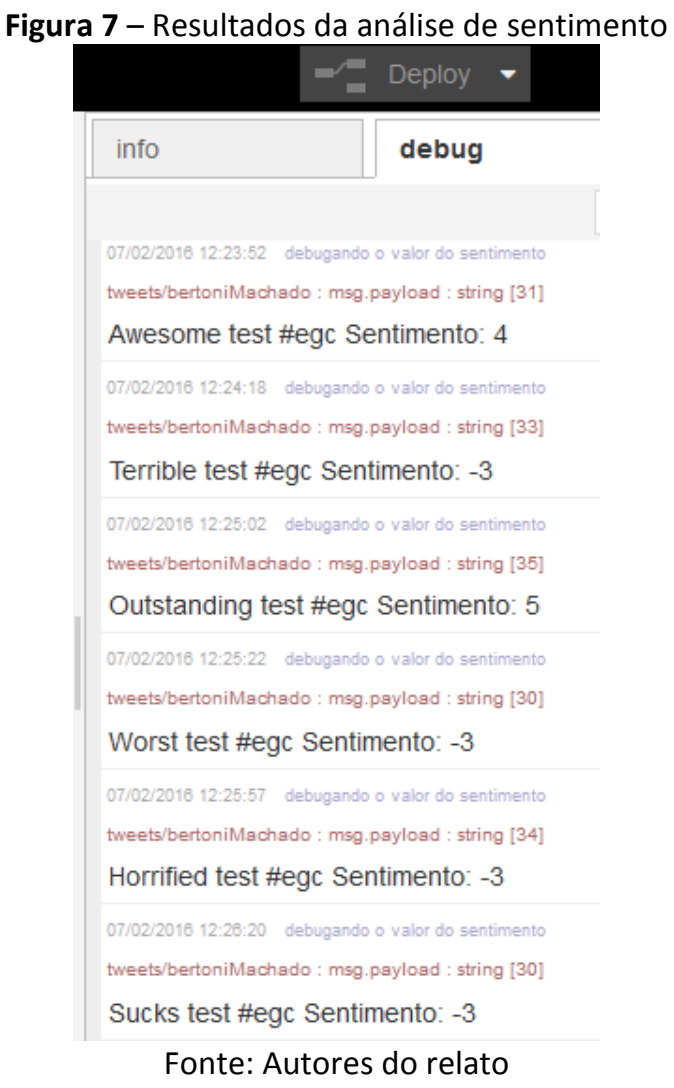

A Figura 8 mostra o e-mail enviado caso o resultado tenha sido analisado com um valor de sentimento menor ou igual a -5 : 
Figura 8 - Mail recebido

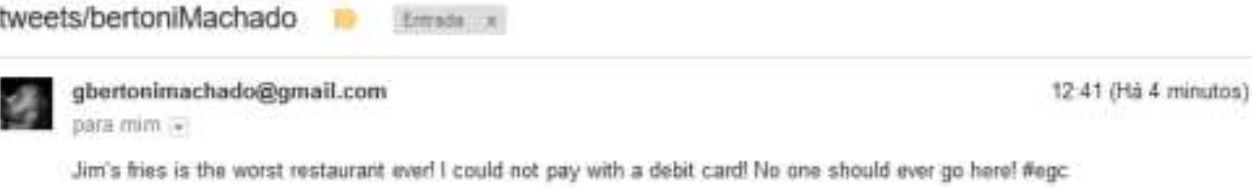

A frase utilizada "Jim's fries is the worst restaurant ever! I could not pay with a debit card! No one should ever go here! \#egc" demonstra que o usuário está muito descontente com o restaurante do Jim, em especial que o serviço de cartão de crédito não funcionou ou não era ofertado. Sabendo disso o dono do estabelecimento pode agir sobre esta demanda.

\section{CONSIDERAÇÕES FINAIS}

Vários temas são importantes para auxiliar a inovação, entre eles a Inteligência Estratégica Antecipativa e Coletiva. O cenário de uso que foi aplicado para a demonstração da análise de sentimento, técnica de Advanced Analytics, tem o intuito de trazer uma forma inovadora para a IEAc, bem como ilustra o processo de forma didática, permitindo a replicação do processo em cenários diferentes.

Neste relato de experiência foi descrito o processo de implantação de uma solução baseada em análise de sentimento para IEAc que tem como propósito servir como ser um cenário didático para saber se as ações e objetivos propostos pela IEAc podem ser alcançados por meio de técnicas de Advanced Analytics.

A utilização do Node-RED se mostrou bastante intuitiva, o monitoramento de posts no Twitter sobre um determinado assunto indexado através da hashtag \#egc foi realizado com poucos passos, bem como a utilização do algoritmo de análise de sentimento. $O$ envio de um e-mail para um determinado usuário contendo o post com uma percepção negativa do assunto monitorado foi recebido praticamente em tempo real, possibilitando que o gestor do estabelecimento (dono da caixa de e-mail) faça a gestão/tomada de decisão sobre o assunto de forma ágil.

Certamente a aplicação da IEAc pode ser um ótimo recurso para uma aproximação aos processos de inteligência das organizações, estimulando a inovação e a pró-atividade.

\section{REFERÊNCIAS}

BAUTZER, Deise. Inovação: repensando as organizações. Editora Atlas SA, 2000.

BENBASAT, Izak; GOLDSTEIN, David K.; MEAD, Melissa. The case research strategy in studies of information systems. MIS quarterly, p. 369-386, 1987.

BOSE, Ranjit. Advanced analytics: opportunities and challenges. Industrial Management \& Data Systems, v. 109, n. 2, p. 155-172, 2009.

BOSE, Ranjit. Competitive intelligence process and tools for intelligence analysis. Industrial Management \& Data Systems, v. 108, Iss. 4, p.510-528. 2008. 
CHEN, Hsinchun; CHIANG, Roger HL; STOREY, Veda C. Business Intelligence and Analytics: From Big Data to Big Impact. MIS quarterly, v. 36, n. 4, p. 1165-1188, 2012.

DAVENPORT, Thomas H. Competing on analytics. harvard business review, v. 84, n. 1 , p. 98 , 2006.

DOS SANTOS, Márcio Carneiro. Inteligência híbrida e análise de sentimentos: integrando curadoria humana e coleta de dados automatizada para avaliar a comunicação de governo. Conexão-Comunicação e Cultura, v. 17, n. 33, 2018.

FELDMAN, Ronen. Techniques and applications for sentiment analysis. Communications of the ACM, v. 56, n. 4, p. 82-89, 2013.

HANSEN, Lars Kai; ARVIDSSON, Adam; NIELSEN, Finn Årup; COLLEONI, Elanor; ETTER, Michael. "Good Friends, Bad News - Affect and Virality in Twitter", The 2011 International Workshop on Social Computing, Network, and Services (SocialComNet 2011), 2011.

JANISSEK-MUNIZ, Raquel; LESCA, Humbert; FREITAS, Henrique. Desenvolvimento da capacidade de antecipação pela identificação e captação de indícios antecipativos em contexto de Inteligência Estratégica Antecipativa. In: CONGRESSO DO IFBAE. 2007.

JANISSEK-MUNIZ, Raquel; LESCA, Humbert; FREITAS, Henrique. Inteligência estratégica antecipativa e coletiva para tomada de decisão. Revista Inteligência Competitiva, v. 1, n. 1, p. 102-127, 2011.

KAHANER, Larry. Competitive intelligence: how to gather analyze and use information to move your business to the top. Simon and Schuster, 1997.

LESCA, H. Inteligência estratégica antecipativa e coletiva: o método LE SCAnning ${ }^{\circledR} /$ Humbert Lesca, Raquel Janissek-Muniz. Porto Alegre: Pallotti, 2015.

LIM, Ee-Peng; CHEN, Hsinchun; CHEN, Guoqing. Business intelligence and analytics: Research directions. ACM Transactions on Management Information Systems (TMIS), v. 3, n. 4, p. 17, 2013.

MANUAL DE OSLO. Proposta de diretrizes para coleta e interpretação de dados sobre inovação tecnológica. OCDE. Organização para Cooperação e Desenvolvimento Econômico: Departamento Estatístico da Comunidade Européia, 2004.

MORIS, Langdon. Permanent innovation: the definitive guide to the principles, strategies, and methods of successful innovators. 2009.

OLIVEIRA, Mírian; MAÇADA, Antonio Carlos Gastaud; GOLDONI, Vanessa. Análise da aplicação do método estudo de caso na área de sistemas de informação. In: Encontro Nacional dos Programas de Pós-Graduação em Administração, 30., Salvador, 2006.

POPADIUK, Silvio; CHOO, Chun Wei. Innovation and knowledge creation: How are these concepts related? International journal of information management, v. 26, n. 4, p. 302-312, 2006. 
QUINTANE, E.; CASSELMAN, R.M.; REICHE, B. S.; NYLUND, P. A. Innovation as a knowledgebased outcome. Journal of Knowledge Management, v. 15, n. 6, p. 928-47, 2011.

SCHUMPETER, Joseph Alois. Teoria do desenvolvimento econômico. Fundo de Cultura, 1961.

SILVA, Nelson Rocha; LIMA, Diego; BARROS, Flávia. Sapair: Um processo de análise de sentimento no nível de característica. In: INTERNATIONAL WORKSHOP ON WEB AND TEXT INTELLIGENCE, 4nd., Curitiba. 2012.

THIOLLENT, Michel. Metodologia da pesquisa-ação. In: Metodologia da pesquisa-ação. Cortez, 2011.

TIDD, Joe; BESSANT, John; PAVITT, Keith. Gestão da Inovação. 3. ed. Artmed Editora, 2008.

TROTT, Paul. Gestão da inovação e desenvolvimento de novos produtos. Porto Alegre: Bookman, 2012.

TYSON, Kirk WM. The complete guide to competitive intelligence: gathering, analyzing and using competitive intelligence. Chicago: Kirk Tyson International, 1998.

XU, J.; HOUSSIN, R.; CAILLAUD, E.; GARDONI, M. Macro process of knowledge management for continuous innovation. Journal of Knowledge Management, v. 14, n. 4, p. 573-591, 2010.

Artigo recebido em 20/05/2017 e aceito para publicação em 01/04/2020 